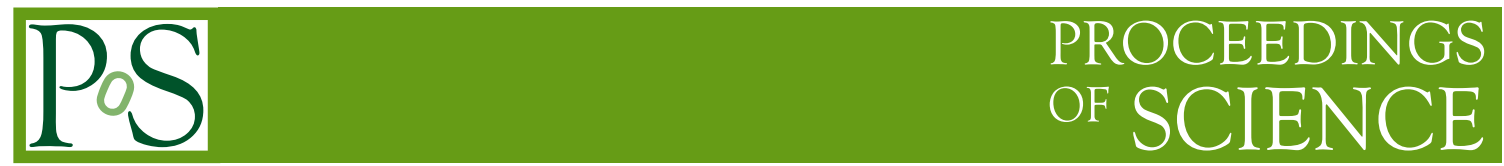

\title{
A deep sea detection prototype
}

\author{
Robert Bormuth On behalf of the KM3NeT Collaboration* \\ Leiden University and Nikhef \\ E-mail: rbormuth@nikhef.nl
}

KM3NeT is a second generation neutrino telescope to be constructed in the Mediterranean Sea. The deployment of the first detection unit prototype in May 2015 off the Italian shore was an important step for the experiment. Not only the production, but also the data analysis allowed for important insights. The powerful options of the multi PMT designs for background rejection and in-situ calibration could be proven. An almost background free sample of muon signals could beidentified and reconstructed. These are important milestones for the KM3NeT experiment leading up its the construction and operation.

XVI International Workshop on Neutrino Telescopes,

2-6 March 2015

Palazzo Franchetti âĂŞ Istituto Veneto, Venice, Italy

${ }^{*}$ Speaker. 


\section{The KM3NeT telescope}

$\mathrm{KM} 3 \mathrm{NeT}$ is a second generation neutrino telescope to be constructed in the Mediterranean Sea. For KM3NeT 2.0 two detectors are planned: ORCA for low energies and ARCA for high energies. The layouts for these two detector types are different, depending on their respective purposes, while sharing the same technology. The KM3NeT telescope will be subdivided into building blocks each consisting of 115 string-type detection units holding 18 digital optical modules (DOM). Each DOM holds 31 photo multiplier tubes (PMT) approximately equally distributed in theta and phi, see Figure 1. The goal of ARCA is the observation of cosmic neutrinos. ORCA focuses on the observation of atmospheric neutrinos in order to measure the unknown mass hierarchy. For more information on the ARCA design and goals see [1] and for ORCA see [2] . Previous to the here discussed string prototype a DOM prototype has been deployed in November 2012 at the site of the predecessor Antares experiment, see [3].

\section{The Prototype}

A first string prototype (PPM-DU) with three instrumented DOMs was deployed on the coast of Sicily in May 2014 at a depth of $3457 \mathrm{~m}$. The string is shortened with respect to a full $18 \mathrm{DOM}$ ARCA string and has a total length of $159.3 \mathrm{~m}$. The three DOMs attached to the string are equipped with PMTs from different producers. The top DOM (DOM 3) holds Hamamatsu PMTs of the type R12199-2 (cathode area 80mm) while the other two hold ETEL PMTs of the type D783KFL (cathode area $77 \mathrm{~mm}$ ). The cathode of each PMT is surrounded by an reflectively coated aluminum ring. The reflector rings of DOM 3 have a larger diameter, causing together with the larger cathode area an increase in total cathode area with respect to the other two DOMs of around 20 percent. Due to the lack of a fully automatized DAQ system prototype data taking was not continuous. A total of $680 \mathrm{~h}$ of data have been recorded since deployment and data taking is still ongoing. A picture of the prototype can be seen in Figure 1. Although the goal of KM3NeT will be the detection of neutrinos, due to the low statistics the PPM-DU focuses on the detection of the far more abundant muons produced in interaction in the earth atmosphere. In order to be able to track these muons, a nano-second precision time calibration of DOMs and PMTs is required.

\section{Time Calibration}

The relative PMT time calibration is performed on each DOM by using the light originating from Potassium decays in the sea salt. The correlated signals from these decays on different PMTs allow for a global fit from which the relative time offsets and efficiencies can be determined. Since neighboring PMTs have more correlated light from Potassium decays than PMTs on opposite sides of the DOM this also allows to check the PMT positioning. The stability of this method has been tested over the course of the full data taking period and was found to be stable within nano second precision.

Once a PMT time calibration for the DOMs is established, the total time offsets between DOMs can be measured. The DOM time calibration is performed by using LEDs installed on the top of each DOM illuminating the DOM above it. By operating the LEDs in special data runs the relative 


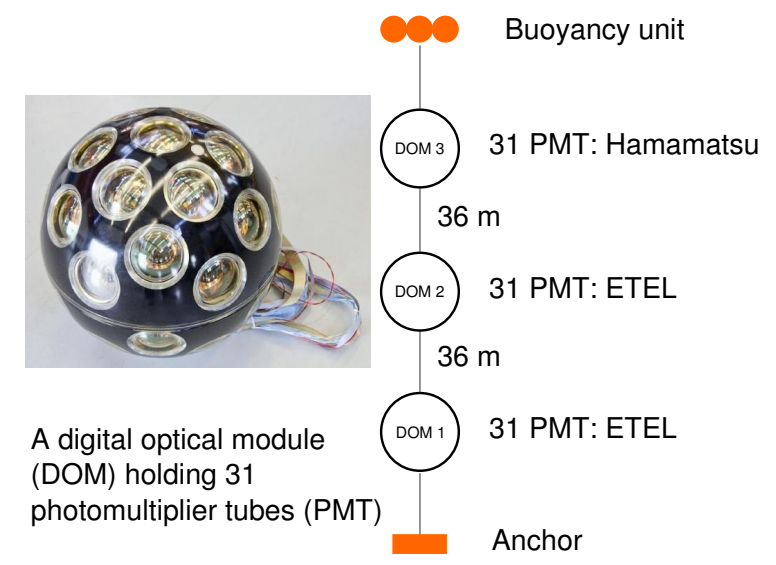

Figure 1: A sketch of a DOM and the PPM-DU

signal travel time differences between the 3 DOMs is determined. It is assumed that the light travels in a straight line from the LED to the PMT of the DOM above. Via a statistical analysis of the time difference in signals in the DOMs the relative time offset can be determined. The stability of the LED calibration has been tested on all recorded LED runs and was found to be stable within 3ns over a period of months.

\section{Muon Signals}

The KM3NeT experiment is a water Cherenkov detector. Charged particles which traverse water at a speed of light faster than the speed of light in water produce a characteristic radiation. This radiation is called Cherenkov radiation and has a determined emission angle and wavelength. These specific characteristics allow for a reconstruction of the charged particles. In order to detect atmospheric muon signals they have to be distinguish from the different background events. The two main backgrounds are Potassium decays and bioluminescence bursts. Both can be distinguished from atmospheric muons by selecting time coincident light hits on the DOMs. By selecting events with at least two hits within 25ns (L1 hit) on each DOM a almost background free atmospheric muon sample can be obtained. In order to verify the observation of muon tracks different properties can be studied. In Figure 2 the zenith orientation of hit PMTs of all three DOMs for atmospheric muon events is shown. A per DOM cut of at least 8 hits within $20 \mathrm{~ns}$ has been applied. For all DOMs upward looking PMTs (along the line) see significantly more light than downward looking PMTs (towards the sea bed). This is consistent with the hypothesis of observing atmospheric muons, since these will hit the detector from above and therefore cause more light in the upward looking PMTs. The higher counts for DOM 3 are caused by the increase in effective collection area as described in Section 2.

\section{Muon Reconstruction}

A simple approach for muon tracking allows for the reconstruction of angular information of the atmospheric muons. By using only the timing information of every first hit on a DOM, a good 


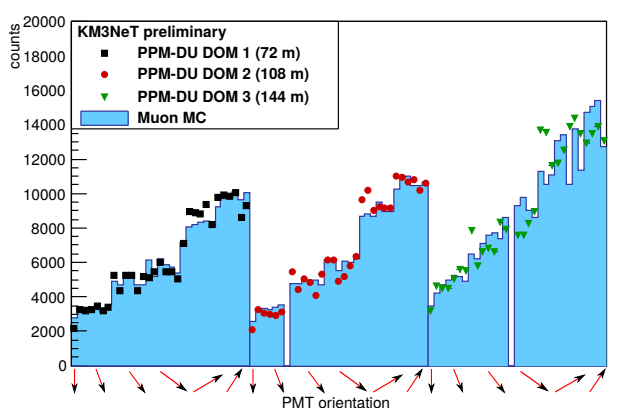

Figure 2: Zenith distribution of hit PMTs for events with an L1 in each DOM

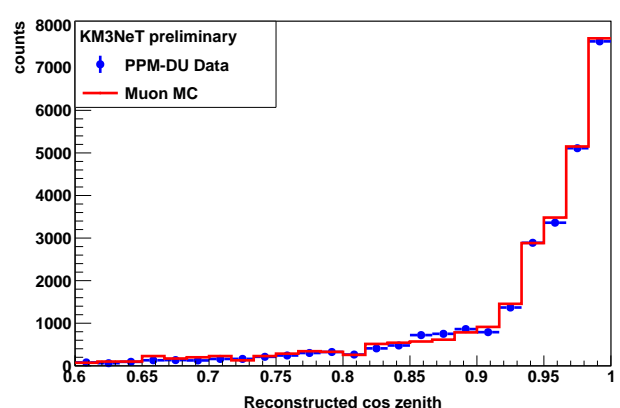

Figure 3: Reconstructed muon zenith compared with simulated muon zenith

zenith reconstruction can be obtained. The zenith angle of a muon track assuming Cherenkov light production can be determined using the following formula:

$$
t=\left[\left(z-z_{0}\right) * \cos \theta+\sqrt{n^{2}-1} * \sqrt{d_{0}^{2}+\left(z-z_{0}\right)^{2} *\left(\sin ^{2} \theta\right)}\right] / c+t_{0}
$$

where $t_{0}, z_{0}, d_{0}$ are the time, height and distance of the closest point between DOM and track, $n$ is the refractive index of light in water, $\theta$ is the zenith angle of the track and $t$ is the expected hit time on the DOM. Since we have 3 measurements of hit times at the 3 DOMs but 4 parameters to fit there are degeneracies in the fit. Therefore causing degeneracies in the phase space. By selecting a part of the phase space with low degeneracies an angular resolution of \pm 3.3 degrees is possible for $70 \%$ of the atmospheric muon events. Figure 3 shows the distribution of the cosine of the zenith for an atmospheric muon simulation and the taken data where excellent agreement can be seen.

\section{Summary}

The first string prototype delivered in depth results on the performances of a KM3NeT detection unit. It allowed to establish methods for in-situ calibration of the future KM3NeT detectors. By exploiting the multi PMT design a nano second accurate time calibration and background rejection are possible. Although only 3 DOMs where deployed, already a good zenith angle reconstruction of atmospheric muons was achieved. The KM3NeT collaboration is working towards the deployment of the first fully functional string in 2015.

\section{References}

[1] KM3NeT Technical Design Report [ISBN 978-90- 6488-033-9];

[2] Katz,U.F. The ORCA Option for KM3NeT 2014: arXiv:1402.1022

[3] Adrián-Martínez, S. et al. Deep sea tests of a prototype of the KM3NeT digital optical module 2014: The European Physical Journal C. 